\title{
Diabetes mellitus in Myanmar: Socio-cultural challenges and strength
}

\author{
Than Than Aye, Moe Wint Aung ${ }^{1}$, Ei Sandar Oo ${ }^{2}$ \\ Department of Medicine, North Okkalapa General Hospital, North Okkalapa, Departments of 'Medicine, and 'Preventive and Social Medicine, \\ University of Medicine 2, Yangon, Myanmar
}

\section{A B S T R A C T}

\begin{abstract}
Myanmar is a country with significant administrative and natural divisions. Access to health-care in few parts is adversely affected by socio-economic as well as infrastructural development. With the prevalence of more than $10 \%$, diabetes needs a special attention including the psycho-social management. Major challenges including public health seeking behavioral issues, presence of traditional medicine, lifestyle and diet and issues pertaining to religion and environmental was studied in the current study to find out ways for improvement in the overall diabetes care in Myanmar.Although, diabetes project, under the guidance and support by World Health Organization, has been implementing the strategies for prevention, early case detection and proper diabetes care in Myanmar, few areas of concern discussed in the paper needs to be taken into consideration and implemented in the project to have the optimal outcome. Not only stake holders but also health-care personals are responsible for improvement in all aspects of diabetes by modification of health-care system and facilitating the desired changes.
\end{abstract}

Key words: Access to health, diabetes prevalence, life-style and diet, socio-cultural challenges, traditional medicine

\section{INTRODUCTION}

Myanmar is the largest country in mainland South East Asia with a total land area of $676,578 \mathrm{~km}^{2}$. The country is divided administratively into 14 states and divisions. It consists of 69 districts, 330 townships, 82 sub-townships, 396 towns, 3045 wards, 1327 village tracts and 67,285 villages. Myanmar falls into three well marked natural divisions; western hills, the central belt and the Shan plateau on the east with the continuation of highland in the Tanintharyi, southern point of Myanmar. Among those divisions, some of the northern and western areas have little access to health-care services, education and social development program compared to central mainland because of the limitation in transportation. ${ }^{[1]}$

\begin{tabular}{|l|l|}
\hline \multicolumn{2}{|c|}{ Access this article online } \\
\hline Quick Response Code: & Website: \\
\hline & www.joshd.net \\
\hline & \\
\hline
\end{tabular}

The population of Myanmar in 2010-2011 is estimated as 59.78 million with the growth rate of $1.1 \%{ }^{[1]}$ About $70 \%$ of the population resides in the rural areas, where as remaining are urban dwellers. Most of the rural people are poor and uneducated hence there is inequality of social and economic development. The Republic of the Union of Myanmar is made up of 135 national races speaking over 100 languages and dialects. Myanmar ethnic groups are different from each other in their geographic location, language and some cultural markers such as national costumes, traditions, customs and some values. The right prominent ethnic groups are Bama, Chin, Kachin, Kayin, Kaya, Mon, Rakhine and Shan. Multi ethnicity and culture is one of the features of Myanmar people. Multi-ethnicities and cultures lead to various kind of belief in traditional medicine (TM) and treatment seeking behaviors. Myanmar has a long history of rich culture and lively traditions and the way of Myanmar people's life has been highly influenced by its cultural norms, social practices and common belief.

Myanmar is also facing double burden of diseases (communicable and non-communicable diseases) due to the demographic and socio-economic transition that

Corresponding Author: Dr. Than Than Aye, Department of Medicine, North Okkalapa General Hospital, North Okkalapa, Myanmar. E-mail: profthanthanaye@gmail.com 
occurred in recent decades like all developing countries. In Myanmar, the prevalence of diabetes mellitus (DM) is in increasing trend. In Yangon, the capital of Myanmar, overall prevalence of adult onset diabetes in 2003-2004 is $11.8 \%$ (urban prevalence is $13.9 \%$ and rural prevalence is 7.3\%). ${ }^{[2]}$ National STEPS Survey (2009) recorded associated risk factors for diabetes and the prevalence of smoking was $33.6 \%$ in males and $6.1 \%$ in females, the prevalence of hypertension was $31 \%$ in males and $29.3 \%$ in females, the prevalence of overweight was $21.85 \%$ in males and $23.07 \%$ in females, the prevalence of obesity was 4.35 in males and 8.45 in females among the sample population. ${ }^{[3]}$

The first Oxford International Diabetes Summit (2002) recommended psycho-social aspects of DM to be included in an individual country's national guidelines. ${ }^{[4]}$ This recommendation was based on diabetes attitudes, wishes and needs, study that included 5000 people with diabetes and 3000 DM healthcare professionals across 13 countries. ${ }^{[5]}$ Its results showed that $40 \%$ of patients had poor psychological well-being, but only $10 \%$ of those received psychological care. It also stressed that the social support (support from family members, co-workers and friends) play a significant role in improving the patient's sense of wellbeing. Psychological well-being may affect self-management of DM. The facts and information mentioned above have led the authors to believe that socio-culture issues of DM in Myanmar need to be addressed urgently. Some of these issues are presented in the following paragraphs.

In Myanmar, there are four major challenges in diabetes care.

1. Role of diabetes care system in Myanmar and public health seeking behavior.

2. Role of TM.

3. Role of life style and diet.

4. Role of religion and environmental challenges.

\section{ROLE OF DIABETES CARE SYSTEM IN Myanmar and Public Health Seeking BEHAVIOR}

Regarding with diabetes care, not only Diabetologists but also general physicians or internists and general practitioners at the primary health-care levels take part in treating diabetes patients. Endocrine and diabetes department has been established in University of Medicine 2, second biggest medical university in Myanmar in 2012 and as a result there is an expansion of diabetes care as well as research work on various endocrine diseases. Well-equipped diabetes clinics of international standards are still need to be developed in every divisions of the country. Since 1991, World Health Organization (WHO) diabetes project for prevention of diabetes and improvement of diabetes care has been established in Myanmar. Apart from that, Myanmar Society of Endocrinology and Metabolism under the Myanmar Medical Association has been established since 2008. Through this society many Continuing Medical Education (CME) activities has been giving to the clinicians to update their knowledge of diabetes care. Recently in 2013, Myanmar Diabetes Association was founded and thereby it will be able to enhance more effective diabetes care throughout Myanmar.

However, there is scarcity of well-formed comprehensive diabetes clinic, which covers retinal screening, foot care, dietary counseling and joint care prenatal clinics. Though all the tertiary hospitals have diabetes clinic, many of those clinics are not set up as international standards. There is still a need to refer to specialist hospitals to get various aspects of care so that people find difficult to do regular follow-ups to be able to achieve good compliance.

One of the factors that influence public health seeking behavior is health insurance system that Myanmar has not established yet. As a result, diabetes patients need to spend money out of their pocket for all costs. In fact, standard diabetes care depends much on the socio-economic status of patient. Most Myanmar diabetes patients have to cope with serious financial constraint especially when glucose monitoring becomes essential in some cases: During insulin therapy, suffering from hypoglycemic problems, needs management of complications, etc. This limitation is one of the reasons that many diabetes patients rely on Myanmar TMs. In fact TM (or) complementary and alternative medicine is the only source of health care for rural and urban poor due to its accessibility and affordability. Thus TM plays an important role in the country's health system.

Although the prevalence is high, there is no enough information available regarding treatment seeking behavior in Myanmar. The health-care seeking behavior is still limited to the traditional view of health (i.e., visits to health-care institutions and health care practitioners; bought medication themselves instead of spending the time to attend specialist diabetes clinic). For many urban residents general practitioners are their first contact point, but for rural residents it is the norm for them to go visit a midwife, who is a primary health-care giver in most rural areas, or a traditional medical practitioner depending on the type of illness, preference of individuals or availability 
of health practitioners. These midwives also act as an initial point of referral for sick people to secondary and tertiary medical treatment. One of the cross-sectional study in 2001 was to examine the treatment-seeking behavior of 147 diabetic patients in Hmaw Bi Township, Yangon Region. The place of first contact for taking care in the government health center was (88 respondents, 57.5\%). The person of first contact for taking care was mainly health-assistant (67 respondents, 43.8\%) and the doctors as the first contact for taking care was only (59 respondents, $38.6 \%$ ). Only $11.1 \%$ had knowledge on all facts of compliance to meet the best control of DM. ${ }^{[6]}$

Some research findings in Asia and Africa indicate that more than $80 \%$ of the populations still rely on TM rather than modern medicine for basic health-care. Socio-cultural values and practices heavily loaded on TM that in turn has a huge impact on the outcome of diabetes cares. ${ }^{[7]}$

\section{ROLE OF TM IN MYANMAR}

Traditional treatments have mostly disappeared in occidental societies, but some are prescribed by practitioners of alternative medicine or taken by patients as supplements to conventional therapy. However, plant remedies are the mainstay of treatment in underdeveloped regions. ${ }^{[8]}$ The traditional plant medicine is used throughout the world for a range of diabetes symptoms. The Papyrus Ebers of $1550 \mathrm{BC}$ recommended a high-fiber diet of wheat grains and ochre. ${ }^{[9]}$ A multitude of herbs, spices and other plant materials have been described for the treatment of diabetes for many decades. ${ }^{[10]}$

In Myanmar, TM is being popular since ancient kingdom dynasty in treating diseases. People belief in plant and herbal medicine put far more weight than standard western medicine not only in minor ailments, but also in major diseases. According to the close group preliminary survey which was done in diabetes clinic of Yangon General Hospital, about 70\% of patients take TM before getting to tertiary clinic. In Myanmar, the usage of TM is expanded with advertisements in media by giving false information that diabetes can be cured totally by their medicine. The traditional practitioners used to say that there is always a cure for every disease. As diabetes is a chronic non-communicable disease the socio economic impact is quite enormous so that the majority of patients want to try the TM instead of standard diabetic treatment because of false belief and low cost.

Renewed attention to alternative medicines and natural therapies has stimulated a new wave of research interest in traditional practices and the WHO expert committee on diabetes has listed as one of its recommendations that traditional methods of treatment for diabetes should be further investigated..$^{[11]}$

University of TM is also developed to upgrade the role of traditional healers. There are also 14 TM hospitals run by the government to supplement numerous TM clinics operated by individuals across the country (Myanmar health-care system 2011). There is also guidance to the research development on Myanmar TM, many research papers appear in traditional health symposium; however, majority are not randomized control trials. Nevertheless this would engender evidence-based research, which would in turn lead to permit incorporation of traditional healers into the health care system. The government encourages the manufacturers to develop TM systematically up to international standards by carrying out both animal and human research to make advertisements upon scientific evidenced based medicine.

In Myanmar, one and famous organic and natural medicine production company has been developed since 2003. This industry has done ample of scientific researches on herbal medicine and found to have promising outcome and gained collaboration not only from regional South East Asian countries, but also from European countries. ${ }^{[12]}$ Regarding with diabetes herbal remedies, the first developed medicines in Myanmar is Java herb, made from Orthosiphon aristatus (Tha-GyarMagike leaves), which is found to have hypoglycemic and antihypertensive effects, the other one is Diabe-herb, made from fruit named Momordica charantia (karela). ${ }^{[13]}$ The study by Myint (2000), showed that the use of SeeCho-Pin/Tha-Gyar-Magike leaves (O. aristatus) in DM patients has helped reduce the fasting blood sugar levels significantly and improved control of diabetes. ${ }^{[14]}$ The study has also pointed out there is no untoward effects occurred in both mice and healthy volunteers.

Both modern and TMs are practiced independently. Gradual co-operation with traditional healers as recommended by the $\mathrm{WHO}$ is perhaps the most promising policy. Mutual respect between care providers in both fields traditional and modern is a pre-requisite for this approach. ${ }^{[9]}$

Although some practices of traditional healers may have negative consequences and it may still not be equal substitute to alternative or modern medical treatment they may be potential partners in the fight against DM, provided that their knowledge and skills are properly recognized and harnessed. 


\section{ROLE OF LIFE-STYLE AND DIET}

\section{Life-style of Myanmar people}

It can be assumed that rural people and city laborers possess high level of physical activities because of their nature of work. These farmers, laborers, fishermen, construction workers do not need special program for physical exercise or sports to keep themselves fit. Most city residents are more likely to have low level of physical activities due to various reasons: No time and no place for doing exercise, sedentary life-style, etc. Not only professional man and women in cities, but also house wives and children find no time for exercise due to increasing screen time watching TV or surfing the Internet and sitting long hours in their car while travelling through the heavy traffic daily. According to the non-communicable disease risk factor survey in Myanmar (National STEPS Survey 2009) male has $10 \%$ - low, $27 \%$ - moderate and $63 \%$ - high-level of physical activity. As for women, the level of physical activity was low in 14\%, moderate in 33\% and high in $53.3 \%$. The mean duration of total physical activity on an average per day was $182 \mathrm{~min}$ (males 211 and females 164). On an average, work related activity comprised a little more than half of the total physical activity among male respondents, while transport activity formed $31.0 \%$ and recreation-related activity comprised $7.9 \%$. For women, work-related activity comprised nearly $60 \%$ of physical activity and transport related activity comprised about $32 \%$. The current smoking is $16.68 \%$ (male $33.61 \%$ and female $6.13 \%$ ), current drinking alcohol is $12.87 \%$ (male $31.17 \%$ and female $1.47 \%$ ). ${ }^{[3]}$

\section{Diet related issues}

Like other nationalities, Myanmar people are fond of eating rich, creamy and delicious food without being aware of the danger of overweight and related health hazards. Besides, they have a positive attitude toward being fat (or) over weight and there is a popular Myanmar saying that "Wa Loe Hla Loe," which means "fat and beautiful", for them fatnesses the symbol of good health and wealth. Nevertheless many of educated people are well aware of the hazards of obesity.

A typical Myanmar meal consists of a lot of rice with some amount of fish or meat, some vegetables (raw or boiled), soup and Nga Pi. Since Nga Pi which is a Myanmar traditional food, come from fish/shrimp preserved with salt, it is very salty. This eating habit can pose more health threat to low-socio-economic groups because they can afford to eat little amount of meat/fish and eat more Nga Pi.
Myanmar common cooking style is not favorable for DM patients Myanmar people tend to cook meat or fish with a lot of oil and spices like Indian style curry or deep fried in peanut oil or palm oil. Myanmar people rarely use butter in their daily cooking. Another good thing about Myanmar diet is a habit of eating plenty of vegetables (raw, boiled and steamed) or mixed with little amount of meat/fish/shrimp.

White rice, being a staple food of Myanmar people, has raised another issue. In general, Myanmar people eat three meals a day (breakfast, lunch and dinner) and in each meal rice is the main part that takes about $75 \%$ of their daily intakes. Widely consumed snacks and desserts in both rural and urban areas are mostly made from rice or glutinous rice; some of them are even mixed with cooked coconut oil or shredded coconut meat.

Myanmar people from big cities are taking up new eating habits due to urbanization and globalization. City dwellers eat more fast food, drink more soft drinks or alcoholic beverages than they used to another factor that makes DM fruits such as banana, mango, durian etc. These fruits are very sweet with high glycemic index. It is not surprising that DM control become more difficult during the rainy season where there are plenty of mangos in the market.

One of the Myanmar favorite traditional foods is tea leaves, they drink black or green tea from dried tea leaves and eat pickled tea leaves mixed with various toasted beans and sesame seeds. Although antioxidants in the tea leaves may be good for health, Myanmar people need to put less oil to the pickled tea leaves.

\section{ROLE OF RELIGION AND ENVIRONMENTAL Challenges}

The majority of its citizens are Buddhists, but there are some communities from other religious groups: Christian, Islam, Hindu, Nats (or) Spirit worshippers. The theme of Buddhism is sacrifice, patience, sharing and forgiving. As a result of Buddhist teaching, one can understand the nature of disease and how to withstand the consequences of chronic diseases. Furthermore, because of the religious teaching and cultural concepts, people understand how to control the grief and how to face the complications of diabetes. Most of Myanmar people use stress management by meditation, taking part in community services and listening to Buddha teachings adapting themselves into chronic diseases.

The Buddhist monks and nuns have a role to influence socio-cultural changes in diabetes management. In rural 
areas, both Buddhist monks and other religious leaders play a leadership role in community by guiding people where to seek medical treatment and how to manage to get funds and donations for the charity clinics. Nowadays, charity clinics are popular in Myanmar especially in poor areas so people get easy access to the western medicine and attain standard medical treatment for diabetes.

The special risk factor related to socio-cultural issue in Myanmar is diabetes foot. Many farmers work bare foot and almost all Myanmar people stay home barefoot making them vulnerable to minor injuries and risk of diabetes foot. One of the common rites and rituals of Myanmar Buddhist people is taking off their foot wears when they visit religious sites: Pagodas, temples, monasteries. Visiting these places during day time, especially at noon, is the common cause of diabetes foot, blister and burns. In some rural and remote areas travelling in an old used cars with poor engine also cause diabetes foot ulcers to those passengers who sit at the front seat close to the hot engine. Most people with foot ulcers do not seek proper treatment; instead they go to traditional healers. They end-up putting all sorts of herbs or leaves or powder of herbs into the ulcers or wounds'. Most of them come to the hospital with late stage with septicemia or gangrene requiring amputation.

\section{CONCLUSION}

It is obvious that efficient diabetic care requires more than world class research and provision of medicines. Many people living with diabetes in Myanmar are still experience ineffective diabetes care due to various socio-cultural as well as psychosocial problems. Although diabetes project, under the guidance and support by WHO, has been implementing the strategies for prevention, early case detection and proper diabetes care in Myanmar there are still many areas to improve to fulfill the needs of all people across the country. After establishing the Myanmar diabetes association, there is a hope for becoming a member of International Diabetes Federation. This will enhance a great move to improve of diabetes care by dissemination of knowledge regarding diabetes among people, as well as improving the standard of diabetes care of health care providers.

Stake holders who are responsible for modification of health-care system in Myanmar need to aware of the impact of the burden of diabetes, there by more attentions can be given to improve all aspects of diabetes care in Myanmar.

\section{REFERENCES}

1. SEARO/Health in Myanmar, 2012. Available from: http://www. searo.who.int/myanmar/documents/healthinmyanmar2012/en/ index.html. [Last accessed on 2013 Aug 8].

2. Soe P, Latt TS, Aung PP, Myint TK. Glucose intolerance and associated factors in 4 townships of Yangon division. STEP Survey, DMR Research Congress, 2004.

3. Non-communicable disease risk factor survey Myanmar 2009. Available from: http://www.who.int/chp/steps/2009_STEPS_ Survey_Myanmar.pdf. [Last accessed on 2013 Aug 8].

4. Anonymous. The Oxford International Diabetes summit: implications of the DAWN study. Pract Diabetes Int 2002;19:187-92.

5. Peyrot M, Rubin RR, Lauritzen T, Snoek FJ, Matthews DR, Skovlund SE. Psychosocial problems and barriers to improved diabetes management: Results of the cross-national diabetes attitudes, wishes and needs (DAWN) study. Diabet Med 2005;22:1379-85.

6. Latt TS, Tun S, Ei Sandar U. Treatment seeking behavior of diabetes mellitus patients in Hmaw Bi Township, Yangon Division; Myanmar. The First International Public Health Conference in Myanmar, 2013.

7. National Policy on Traditional Medicine and Regulation of Herbal Medicines: Report of a Global WHO Survey. Geneva: WHO; 2005.

8. Bailey CJ, Day C. Traditional plant medicines as treatments for diabetes. Diabetes Care 1989;12:553-64.

9. The Papyrus Ebers: The Greatest Egyptian Medical Document. Copenhagen: Levin and Munksgaard; 1937.

10. Ajgaonkar SS. Herbal drugs in the treatment of diabetes: A review. Int Dairy Fed Bull 1979;24:10-7.

11. Chinnenye $\mathrm{S}$, Ogbera $\mathrm{AO}$. Socio-cultural aspects of diabetes mellitus in Nigeria. J Soc Health Diabetes 2013;1:15-21.

12. Fame Pharmaceuticals, Myanmar organic medicine. Available from: http://www.famepharma.com. [Last accessed on 2013 Aug 7].

13. Vad BG. Place of Momordica charantia in the treatment of diabetes mellitus. Maharashtra Med 1960;16:733-45.

14. Myint KY. The prevention and management of type 2 diabetes mellitus in Myanmar. Doctorate Thesis Submitted to Board for Degree of Doctor of Medicine, University of Medicine 2, October, 2000.

How to cite this article: Aye TT, Aung MW, Oo ES. Diabetes mellitus in Myanmar: Socio-cultural challenges and strength. J Soc Health Diabetes 2014;2:9-13.

Source of Support: Nil. Conflict of Interest: None declared. 